\title{
REPRESENTATION AND COMPUTATION OF THE PSEUDOINVERSE ${ }^{1}$
}

\author{
D. SHOWALTER
}

I. Introduction. The theory of pseudoinverses, or generalized inverses, has been extensively developed over the last few years. A recent and comprehensive bibliography occurs in [2] which gives a short history also, and a brief survey of known results and computational methods.

We consider here the case of a bounded operator with closed range between Hilbert spaces. Most of the information for this case, which is used in this paper can be found in [3], which is pithy and short.

II. Notation and basic information. $A$ is a bounded linear transformation with closed range from $S_{1}$ to $S_{2}$, where $S_{1}$ and $S_{2}$ are Hilbert spaces with inner product norms $|\cdot|_{1}$ and $|\cdot|_{2}$, respectively. $A^{*}$ is the adjoint of $A, A^{\dagger}$ is the pseudoinverse of $A$ (uniquely defined for this case [3]). $I_{1}$ and $I_{2}$ are the identity mappings on $S_{1}$ and $S_{2}$, respectively. $\bar{S}_{1}=A * S_{2}=A * A S_{1}$ and $\bar{S}_{2}=A S_{1}=A A^{*} S_{2}$. We use a bar to denote the restriction of a mapping to $\bar{S}_{1}$ or $\bar{S}_{2}$, as appropriate, e.g., $\bar{A}=A \mid \bar{s}_{1}$ the restriction of $A$ to $\bar{S}_{1}$. We know that $(\bar{A})^{-1}$ exists and is bounded [3]; and it is readily deduced that $\left|A^{\dagger}\right|=\left|(\bar{A})^{-1}\right|$ (from [3, Theorem 3], noting that $\bar{S}_{1}=\mathfrak{M}(A)^{\perp}$, and $\left.\bar{A}^{\dagger}-(\bar{A})^{-1}=0\right)$. Thus, we know a priori that $A^{\dagger}$ is a uniquely defined, bounded linear transformation from $S_{2}$ to $S_{1}$. We use the conventional norms on linear transformations. We use the basic definition of the pseudoinverse given by Desoer and Whalen [3], for the case studied $B=A^{\dagger}$ if and only if $B$ is a linear operator such that

(i) $B \bar{A}=\bar{I}_{1}$,

(ii) $B \bar{S}_{2}^{\perp}=0$ (i.e., $B y=0$ if $y$ is in the orthogonal complement of $\bar{S}_{2}$ ). From this definition it follows that $A A^{\dagger} A=A$ and $A^{*} A A^{\dagger}=A^{*}$ $=A^{\dagger} A A^{*}$, since $S_{1}=\bar{S}_{1} \oplus \bar{S}_{1}^{\perp}$ and $S_{2}=\bar{S}_{2} \oplus \bar{S}_{2}^{\perp}$.

III. TheOREM 1. Define, for $t \geqq 0$,

$$
A^{\dagger}(t)=\int_{0}^{t} \exp \left[-A^{*} A(t-s)\right] A^{*} d s
$$

Received by the editors July 7, 1966.

1 This paper represents results obtained at the Courant Institute of Mathematical Sciences, New York University, with the National Science Foundation, Grant NSF-GP-3465. 
Then

$$
\left|A^{\dagger}-A^{\dagger}(t)\right| \leqq\left|A^{\dagger}\right| \exp \left[-t\left|A^{\dagger}\right|^{-2}\right], \quad \forall t .
$$

Proof. Since $A^{*}=A^{*} A A^{\dagger}$, (3.1) may be evaluated as: $A^{\dagger}(t)$ $=\left[I_{1}-\exp \left[-t A^{*} A\right]\right] A^{\dagger}$. Pick $y \in S_{2}$, and let $\alpha(t)=\left[A^{\dagger}-A^{\dagger}(t)\right] y$ $=\exp \left[-t A^{*} A\right] A^{\dagger} y$. Clearly, $\alpha(t) \in \bar{S}_{1}, \forall t$. Thus $d|\alpha(t)|_{1}^{2} / d t=$ $-2|A \alpha(t)|_{1}^{2}$ (see $\left.[5]\right) \leqq-2\left|A^{\dagger}\right|-2|\alpha(t)|^{2}$, whence $\left|A^{\dagger} y-A^{\dagger}(t) y\right|$ $\leqq\left|A^{\dagger} y\right| \exp \left[-t\left|A^{\dagger}\right|^{-2}\right]$, and (3.2) follows since $y$ is arbitrary.

The next theorem has been proved for the matrix case by den Broeder and Charnes (see [2]), and for the nonsingular case by Altman [1].

Theorem 2. Pick c so that $0<c<2, \forall n \geqq 0$, define

$$
B_{n, c}=\sum_{p=0}^{n}\left[I_{1}-\frac{c A^{*} A}{|A|^{2}}\right]^{p} \frac{c A^{*}}{|A|^{2}} .
$$

Define:

$$
\beta_{c}=\max \left\{|1-c|,\left.|1-c /| A\right|^{2}\left|A^{\dagger}\right| 2 \mid\right\}<1 .
$$

Then

$$
\left|A^{\dagger}-B_{n, c}\right| \leqq\left(c \beta_{c}^{n+1} /\left(1-\beta_{c}\right)\right)|A|, \quad \forall n \geqq 0 .
$$

Proof of Theorem 2. Let $B_{\infty, c}=\lim _{n \rightarrow \infty} B_{n, c}$, and define $E$ $=c A^{*} A /|A|^{2}$. Then $\left|\bar{I}_{1}-\bar{E}\right| \leqq \beta_{c}$ (see $[1$, pp. 52-55]) so that $(\bar{E})^{-1}=\sum_{p=0}^{\infty}\left(\bar{I}_{1}-\bar{E}\right)^{p}$ (where $(\bar{E})^{-1}$ is defined on $\bar{S}_{1}$, the range of $\bar{E}$ ). Now $\bar{E}: \bar{S}_{1} \rightarrow \bar{S}_{1}$ and $\left(I_{1}-E\right): \bar{S}_{1} \rightarrow \bar{S}_{1}$, so

$$
\begin{aligned}
B_{\infty, c} \bar{A} & =\sum_{p=0}^{\infty}\left(I_{1}-E\right)^{p} \bar{E} \\
& =\sum_{p=0}^{\infty}\left(I_{1}-\bar{E}\right)^{p} \bar{E}=\bar{I}_{1} .
\end{aligned}
$$

$B_{n, c} \bar{S}_{2}^{\perp}=0, \forall n$, since $A^{*} \bar{S}_{2}^{\perp}=0$, in view of (3.3). Linearity is obvious. Thus $A^{\dagger}=B_{\infty, c}$; and (3.4) follows immediately, since it is the rate of convergence on $\bar{S}_{2}$ (see $[1$, p. 52-55]). Optimal convergence occurs when

$$
c=2|A|^{2}\left|A^{\dagger}\right|^{2} /\left(|A|^{2}\left|A^{\dagger}\right|^{2}+1\right),
$$

in which case

$$
\beta_{c}=\left(|A|^{2}\left|A^{\dagger}\right|^{2}-1\right) /\left(|A|^{2}\left|A^{\dagger}\right|^{2}+1\right) \quad[1]
$$

which yields the following 
Corollary to Theorem 2. If $|A|=\left|A^{\dagger}\right|=1$, then $A^{\dagger}=A^{*}$.

The rates of convergence in the previous theorems are often impracticably slow. The following theorem gives a rapidly convergent recursion sequence, analogous to a well-known technique for improving estimates of the inverse for a nonsingular matrix [4, p. 120].

Theorem 3. Pick cand define $\beta_{c}$ as in Theorem 2. Define the sequence:

$$
D_{0, c}=c A^{*} /|A|^{2} ; \quad D_{n+1, c}=2 D_{n, c}-D_{n, c} A D_{n, c} .
$$

Then

$$
\left|A^{\dagger}-D_{n, c}\right| \leqq\left|A^{\dagger}\right| \cdot \beta_{c}^{2^{n}} .
$$

Proof of Theorem 3. Define $\Gamma_{c}=\left[A \dagger A-D_{0, c} A\right] . \Gamma_{c} \bar{S}_{1}^{\perp}=0$ and $\bar{\Gamma}_{c}=\bar{I}_{1}-\bar{E}$ (see proof of Theorem 2) so $\left|\Gamma_{c}\right| \leqq \beta_{c}$. By definition $\Gamma_{c}^{2^{0}}=\left[A \dagger A-D_{0, c} A\right]$. Suppose that $\Gamma_{c}^{2^{n}}=\left[A \dagger A-D_{n, c} A\right]$. Then

$$
\begin{aligned}
\Gamma_{c}^{2^{n+1}}=\left(\Gamma_{c}^{2^{2}}\right)^{2}= & A^{\dagger} A A^{\dagger} A-A^{\dagger} A D_{n, c} A \\
& -D_{n, c} A A^{\dagger} A+D_{n, c} A D_{n, c} A \\
= & A^{\dagger} A-\left(2 D_{n, c}-D_{n, c} A D_{n, c}\right) A \\
= & {\left[A^{\dagger} A-D_{n+1, c} A\right], }
\end{aligned}
$$

since $D_{n, c} A: S_{1} \rightarrow \bar{S}_{1}, \forall n$, which is clear from (3.5), and since $A A^{\dagger} A=A$. Also from (3.5), $D_{n, c} \bar{S}_{2}^{\perp}=0=A^{\dagger} \bar{S}_{2}^{\perp}, \forall n$. Since $\left|\Gamma_{c}\right| \leqq \beta_{c}$, and $A^{\dagger}-D_{n, c}=\left(A^{\dagger}-D_{n, c}\right) A A^{\dagger}, \forall n$, (3.6.) follows.

IV. So long as $\left|A^{\dagger}\right|<\infty$, the hypothesis that $|A|<\infty$ may be relaxed in Theorem 1 . We need only require that $A^{*} A$ is a closed mapping with dense domain. The proof as given remains valid (see [5]).

If $A$ fails to have closed range, but is bounded, then $A^{\dagger}(t), B_{n, c}$, and $D_{n, c}$ converge monotonically to $A \dagger$, but (3.2), (3.4), (3.6) are useless since $\left|A^{\dagger}\right|=\infty$ and $\beta_{c}=1$. The associated proofs are more lengthy and will be published elsewhere.

\section{REFERENCES}

1. M. Altman, Approximation methods in functional analysis, Lecture Notes, Ma 107c, California Institute of Technology, Pasadena, 1959.

2. A. Ben Israel and A. Charnes, Contributions to the theory of generalized inverses, SIAM J. 11 (1963), 667-697.

3. C. A. Desoer and B. H. Whalen, A note on pseudoinverses, SIAM J. 11 (1963), 442-447.

4. R. A. Frazer, W. J. Duncan and A. R. Collar, Elementary matrices, Cambridge, London, 1960.

5. T. Kato, Linear differential equations in Banach space, New York Univ. Res. Rep. BR-11, New York, 1955.

Courant Institute of Mathematical Sciences, New York University 